\title{
LAS CONDICIONALIDADES COMO EXPRESIÓN DE LA "INVERSIÓN DE LA DEUDA": transferencias de renta, Capital humano y superación de la pobreza intergeneracional ${ }^{1}$
}

\section{Maria Laura Vecinday Garrido}

Universidad de la República (UDELAR)

\begin{abstract}
LAS CONDICIONALIDADES COMO EXPRESIÓN DE LA "INVERSIÓN DE LA DEUDA": transferencias de renta, Capital humano y superación de la pobreza intergeneracional

Resumen: Las condicionalidades exigidas en los programas de transferencia de renta (PTRC), su fundamento en la "teoría" del capital humano y social como estrategia de "combate" a la pobreza, su significación sociopolítica en el campo social y los mecanismos de gestión asociados a su contralor, ha sido una de las dimensiones abordadas para el análisis de los PTRC en el marco del proyecto Estudio Comparado de los Programas de Transferência de Renda na América Latina. El trabajo presenta los debates sobre el tema con énfasis en los aspectos que constituyen un denominador común identificable en los PTRC analizados.

Palabras clave: Transferencia de renta, condicionalidades, Capital humano.

THE CONDITIONALITIES AS EXPRESSION OF "DEBT INVESTMENT": income transfers, human Capital and overcoming the intergenerational poverty

Abstract: Conditionalities required by income transfer programs (PTRC), founded on the "theory" of human and social capital as a strategy for "combat" poverty, its socio-political significance in social field and the management mechanisms associated with its control, has been one of the dimensions addressed in the analysis of PTRC in the project Comparative Study of Income Transfer Programs in Latin America. The paper presents the debates on the topic with emphasis on those aspects which constitute a common denominator in these PTRC.

Key words: Income transfer, conditionalities, human Capital.
\end{abstract}




\section{INTRODUCCIÓN}

Los Programas de Transferencia de Renta Condicionada (PTRC) vehiculizan un discurso y una práctica apoyados en un supuesto básico: la pobreza es resultado de carencias de capital social presentes en las familias pobres. Este supuesto define la forma de intervención sobre estas familias proponiendo su participación en actividades orientadas a ampliar su capital humano mediante la exigencia de condicionalidades. Las transferencias de renta no representan una novedad en el repertorio de programas sociales. En varios países de la región se encuentran antecedentes de programas con presencia del mecanismo de la transferencia de renta. Lo novedoso es la dimensión que han tomado estos programas, que representan la principal estrategia de combate de la pobreza en la región, la exhaustividad de su presencia en América Latina y que su administración demanda la constitución de instancias institucionales específicas.

El tema de las condicionalidades ha generado importantes debates y controversias en los análisis sobre los PTRC. La problematización de las condicionalidades asociadas a las transferencias de renta ha sido una de la dimensiones seleccionadas para el Estudio Comparado de los Programas de Transferência de Renda na América Latina y es el origen de esta presentación.

El trabajo sintetiza los contenidos centrales que fueron objeto de reflexión en el estudio de las condicionalidades: principales posiciones en el debate, su particular expresión en los tres programas comparados (Bolsa Família - Brasil, Asignación Universal por Hijo - Argentina y Asignación Familiar del Plan de Equidad - Uruguay), su fundamento en la teoría del capital humano, su significación sociopolítica en el campo social y los mecanismos de gestión asociados a su contralor.

Las condicionalidades son parte constitutiva de los PTRC más allá de las particularidades que asume su gestión y contralor en los distintos países. De este modo, el análisis de las condicionalidades representa una puerta de entrada privilegiada para comprender la orientación general que adoptan las politicas de combate o de alivio a la pobreza desplegadas en la región en estas últimas décadas. El análisis de las condicionalidades aporta elementos para comprender cómo los promotores de los PTRC interpretan el fenómeno de la pobreza, a qué factores atribuyen su perpetuación, sobre qué aspectos y hacia dónde se debe orientar la intervención, qué responsabilidades le caben al Estado y cuáles a los individuos, etc. Además, cuando este análisis incorpora las particularidades de los contextos nacionales permite avanzar en la comprensión del papel específico que desarrollan las condicionalidades en cada caso y los distintos matices que adopta su control y seguimiento.

El trabajo avanza, sin pretensión de exhaustividad, sobre algunas derivaciones analíticas que se desprenden del estudio de las condicionalidades presentes en los PTRC estudiados.

\section{LA EXIGENCIA DE CONDICIONALIDADES COMO FORMA DE "COMBATE" A LA POBREZA}

Las condicionalidades no refieren a las condiciones o atributos de elegibilidad que deben satisfacer los potenciales beneficiarios de una prestación sino que son imposiciones que deben cumplir los beneficiarios de una prestación como condición para continuar recibiéndola. Si las operaciones de identificación y selección de la población a la cual se focaliza la prestación nos informan sobre el perfil de los beneficiarios, las condicionalidades nos informan sobre los comportamientos promovidos para esta población.

Del estudio comparado de la Asignación

\section{Cuadro 1 - Condicionalidades sanitarias y educativas por programa analizado}

\begin{tabular}{|c|c|c|c|}
\hline $\mathrm{AUH}$ & $\begin{array}{l}\text { Controles sanitarios y plan de } \\
\text { vacunación obligatorio hasta } \\
\text { los } 4 \text { años }\end{array}$ & $\begin{array}{l}\text { Asistencia a establecimientos } \\
\text { educativos públicos (5 a } 18 \\
\text { años) }\end{array}$ & \\
\hline AFAM-PE & $\begin{array}{|lr|}\text { Controles } & \text { establecidos } \\
\text { embarazadas } & \text { obstétricos para } \\
\text { las }\end{array}$ & $\begin{array}{l}\text { Asistencia establecimientos } \\
\text { educativos de adolescents y a } \\
\text { niños }\end{array}$ & \\
\hline $\mathrm{BF}$ & $\begin{array}{l}\text { Embarazadas: inscripción en } \\
\text { pre-natal y consultas médicas } \\
\text { establecidas } \\
\text { Responsables de niños } \\
\text { menores de } 7 \text { años: participar } \\
\text { de actividades educativas } \\
\text { (amamantamiento materno y } \\
\text { alimentación saludable). } \\
\text { Niños de } 0 \text { a } 7 \text { años: carné de } \\
\text { salud, vacunación }\end{array}$ & $\begin{array}{l}\text { Asistencia minima de } 85 \% \\
\text { en niños y de } 75 \% \text { en } \\
\text { adolescentres entre } 6 \text { y } 15 \text { años } \\
\text { a establecimientos educativos. }\end{array}$ & $\begin{array}{l}\text { Asistencia Social: niños y } \\
\text { adolescentes hasta } 15 \text { años } \\
\text { en situación de riesgo o } \\
\text { retirados del trabajo infantil } \\
\text { deben participar de los } \\
\text { Serviços de Convivência e } \\
\text { Fortalecimento de Vínculos, } \\
\text { con actividades en el } \\
\text { turno libre, alcanzando uma } \\
\text { frecuencia minima de } 85 \%\end{array}$ \\
\hline
\end{tabular}

Fuente: Elaborado pela autora. 
Cuadro 2 - Efectos del incumplimiento y mecanismos de control de condicionalidades por programa analizado

\begin{tabular}{|c|c|c|c|}
\hline $\mathrm{AUH}$ & $\begin{array}{l}\text { La falta de acreditación produce } \\
\text { la pérdida del beneficio. Los } \\
\text { preceptores reciben el } 80 \% \text { del } \\
\text { monto total de la transferencia } \\
\text { y el } 20 \% \text { restante se abona de } \\
\text { forma anual al verificarse el } \\
\text { cumplimiento }\end{array}$ & $\begin{array}{l}\text { Acreditación: mediante registro } \\
\text { de autoridades educativas } \\
\text { y sanitarias en la Libreta } \\
\text { Nacional del niño o adolescente } \\
\text { Organismo: ANSES }\end{array}$ & \\
\hline AFAM-PE & $\begin{array}{l}\text { La falta de acreditación produce } \\
\text { la pérdida del beneficio. }\end{array}$ & $\begin{array}{l}\text { Acreditación: bases de } \\
\text { datos con cruzamiento de } \\
\text { información sobre asistencia } \\
\text { a centros educativos. } \\
\text { No se han controladolas } \\
\text { condicionalidades sanitarias. } \\
\text { Organismo: BPS }\end{array}$ & \\
\hline $\mathrm{BF}$ & $\begin{array}{l}\text { La falta de acreditación } \\
\text { desencadena: } \begin{array}{l}\text { advertencia, } \\
\text { bloqueo, suspensión } \\
\text { cancelación }\end{array}\end{array}$ & 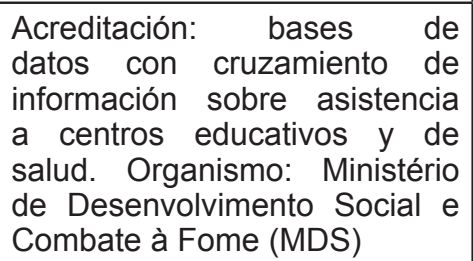 & Seguimiento: municipios \\
\hline
\end{tabular}

Fuente: Elaborado pela autora.

Universal por Hijo (AUH) en Argentina, las Asignaciones Familiares del Plan de Equidad (AFAM-PE) en Uruguay y del Bolsa Familia (BF) en Brasil se desprende que en los tres casos se exigen condicionalidades educativas y sanitarias y que las principales diferencias se registran en los mecanismos de control, verificación y tipo de sanción en caso de incumplimiento. El Cuadro 1 resume las condicionalidades exigidas por cada programa.

De este segundo cuadro, se deduce que los criterios ante situaciones de incumplimiento son difererentes en cada programa:

- En las AFAM-PE el beneficio se pierde;

- En la AUH se retiene una parte de la transferencia hasta que se verifica el requisito de la condicionalidad y se pierde si esto no se constata;

- En el BF el beneficio se retira pero se acumula y reintegra una vez cumplida la condicionalidad.

El debate sobre las condicionalidades puede ser ordenado según tres perspectivas:

a) Las condicionalidades entendidas como forma de acceso y ampliación de derechos es la perspectiva oficial que acompaña la fundamentación del carácter condicionado de la prestación. Las condicionalidades contribuirían para afirmar los derechos sociales básicos.

b) Las condicionalidades entendidas como negación de derechos representa la concepción opuesta a la anterior, por considerar que a un derecho no se le pueden imponer contrapartidas, exigencias o condicionalidades como forma de mantener el beneficio.

c) Las condicionalidades entendidas como mecanismos de control social y moral de los pobres y sus comportamientos. Esta concepción aparece fuertemente representada en un sentido común social que considera legítimas y deseables las condicionalidades en tanto mecanismos de educación de los pobres, reclamando de los gobiernos el control y el castigo ante su incumplimiento (SILVA; GUILHON; LIMA, 2013).

Como fuera dicho, los discursos oficiales registrados en los documentos de los casos analizados sitúan la perspectiva oficial sobre las condicionalidades entendiéndolas como forma de efectivizar derechos sociales en el marco de programas de combate a la pobreza.

Otro rasgo distintivo es que solo el BF prevé acciones dirigidas a identificar e intervenir sobre los factores asociados al no cumplimiento. En estas situaciones, la primer respuesta es incentivar a las familias para el apego a los comportamientos derivados de las condicionalidades hasta llegar a la pérdida del beneficio como sanción. No ocurre los mismo con las AFAM-PE y la AUH que no despliegan intervenciones asociadas al incumplimiento ni advierten a la familia sobre la posibilidad de perder el beneficio.

Esta nueva generación de programas de combate a la pobreza promovida por organismos internacionales y adoptada por los países del continente privilegia la transferencia de renta como principal respuesta al problema. Las transferencias pueden asumir distintas modalidades pero ha sido la transferencia en metálico la opción preferencial.

El problema de las distintas opciones de transferencias (en metálico, en especies o combinadas) ha sido estudiado fundamentalmente 
por la teoría económica registrándose los primeros trabajos sobre el tema en la década del 40. Esta literatura centra sus estudios en si los Estados deben producir bienes y servicios o limitarse a transferir renta de forma que los individuos acudan al mercado para resolver sus demandas. Muchos argumentos en el campo de la teoría económica, preocupados por la utilidad del receptor, apoyan esta alternativa fundándose en que (i) habilita mayor margen de libertad a la acción individual, (ii) podría, eventualmente, resultar menos onerosa para las arcas del Estado y (iii) que nadie conoce mejor sus necesidades que aquel que laspadece. Estos estudios jerarquizan lo que se ha dado en llamar la doctrina de la soberanía absoluta del consumidor (PIEDRA, 2004). Este parece ser el espíritu que orientó el diseño de los PTRC analizados (BENTURA; VECINDAY, 2015).

Sin embargo, estos programas no solo transfieren renta sino que condicionan la continuidad del beneficio al ejercicio de ciertas prácticas. En ese sentido, la doctrina de la soberanía absoluta del consumidor se articula con exigencias comportamentales que responden a una especial preocupación para que la transferencia cumpla con los fines previstos, es decir constituir un estímulo para que sus beneficiarios demanden servicios educativos y sanitarios. Tal preocupación se materializa en el establecimiento de una vigilancia más o menos estricta sobre el cumplimiento de las condicionalidades y supone cierta preocupación por la incompetencia moral de los pobres y su incapacidad de "[...] gobernar el conflicto endémico entre sus deseos y sus capacidades." (BAUMAN, 2001, p. 80-83). La transferencia en dinero sujeta a condicionalidades pretende operar directamente como un estímulo económico para modificar determinados comportamientos de la población beneficiaria siguiendo el supuesto de la racionalidad del homoeconomicus y fundamentado en la idea de que la pobreza y su reproducción intergeneracional también responde a atributos comportamentales de los pobres.

Como vimos, los promotores de los PTRC argumentan que la exigencia de condicionalidades educativas y sanitarias constituye una estrategia de superación intergeneracional de la pobreza. Los fundamentos para la exigencia de condicionalidades en educación y salud se encuentran en la denominada teoría del capital humano. Esta teoría promueve la idea de que la pobreza puede ser superada mediante el desarrollo de las capacidades de los pobres y concluye que la inversión destinada a elevar las capacidades de los pobres constituye una estrategia apropiada para combatir la pobreza. La perpetuación de la pobreza, desde esa perspectiva, podría ser alterada si se superan las ausencias o déficits de capacidades y destrezas que dificultan a los individuos superar su desempeño en el mercado.
La teoría del capital humano es difundida en la década del 90 por el Programa de las Naciones Unidas para el Desarrollo (PNUD) como principios orientadores y recomendaciones para la implementación de medidas estratégicas de lucha contra la pobreza. Su origen data de 1959 con la obra del economista Theodore Schultz quien destaca el papel de la educación y la formación de capacidades como instrumento para mejorar los desempeños productivos.

Más próxima en el tiempo es la obra del también economista Amartya Sen (2000), quien reafirma la idea de las capacidades humanas como factor de desarrollo y superación de la pobreza. La mayor capacidad se correspondería con un mayor poder para obtener ingresos y, agrega que

\section{[...] la mejora de la educación básica y de la asistencia sanitaria no sólo aumenta la calidad de vida directamente sino también la capacidad de una persona para ganar una renta y librarse, asimismo, de la pobreza de renta. (SEN, 2000, p.117-118).}

En igual sentido se orienta Giddens, Beck e Lash (1997) en su crítica a los Estados de Bienestar y su aparente inadecuación a los contextos tardomodernos. Luego de afirmar que "[...] la redistribución directa de la riqueza [...] no sería más que una solución parcial a los problemas de la pobreza." concluye que

\begin{abstract}
[...] ya no es útil concebir algún tipo de gigantesco estado redistributivo del bienestar. Tenemos que pensar en otros términos [...] las medidas adoptadas para contrarrestar el subprivilegio deben ser capacitadoras. (GIDDENS; BECK; LASH, 1997, p. 233-234).
\end{abstract}

Estas perspectivas tienen en común el desplazar el foco del análisis sobre la génesis y reproducción de la pobreza: los problemas de distribución y redistribución son minimizados y, en su lugar, son jerarquizadas estrategias tales como la inversión en capital humano y el fomento de las capacidades individuales. De este modo, el acceso a servicios educativos y sanitarios básicos sería la estrategia fundamental para combatir la pobreza y ha sido una estrategia promovida por los organismos internacionales de crédito en pleno apogeo del modelo neoliberal y hasta nuestros días.

El nacimiento del siglo XXI ha sido testigo del fracaso de estas iniciativas y de la agudización de los efectos sociales regresivos del modelo económico orientado por el proyecto neoliberal. La desigualdad persiste pese a la disminución de la pobreza y la indigencia y a las mejoras en otros indicadores sociales y económicos. En el caso uruguayo, la desigualdad de ingresos se encuentra prácticamente estancada: en 2014 el $20 \%$ de hogares más rico 
se apropió del $42,9 \%$ del total de ingresos del país mientras que al $20 \%$ más pobre le correspondió el $6,9 \%$ del total de ingresos (INSTITUTO CUESTA DUARTE, 2015). Esto refuerza la idea de que es posible reducir la pobreza y la indigencia y, al mismo tiempo, aumentar la desigualdad. Sin embargo, la teoría del capital humano ha sido una sobreviviente que encuentra en los PTRC su reactualización bajo la forma de condicionalidades que acompañan a las transferencias. De este modo, los PTRC apuntan a combatir la pobreza mediante estrategias de corto y largo plazo. La transferencia opera en el alivio inmediato de la pobreza mientras que las condicionalidades son fundamentadas como estrategia a largo plazo para interrumpir el ciclo de reproducción intergeneracional de la pobreza.

La exigencia de condicionalidades tiene la pretensión de incidir en la demanda que los preceptores puedan hacer de los servicios educativos y sanitarios relegando a un segundo plano el problema de la oferta, es decir, la responsabilidad y las capacidades estatales de garantizar el acceso y el tránsito de los usuarios de estos servicios. Centrar el combate a la pobreza en propuestas de intervención ancladas en la demanda es una de las tantas formas de individualización social al privilegiar el papel de las decisiones de los individuos sobre sus comportamientos y estilos de vida para explicar su situación de desventaja.

Hablar de la individualización de la protección es hablar de,

[...] una forma específica de la orientación de la protección social que se caracteriza por atar las condiciones de vida de las personas a la situación y las prácticas, en sentido amplio, del sujeto en tanto individuo; y que, a la vez desvincula esas condiciones de vida y las consecuencias de esas prácticas de toda relación con y de toda pertenencia a grupos o agregados mayores. (DANANI, 2008, p. 45).

Individualizar la protección social es "[...] vincular el otorgamiento de una prestación con la consideración de la situación específica y la conducta personal de los beneficiarios." y ha sido la orientación dominante del nuevo régimen de protección orientado a "[...] los dejados de lado de las protecciones clásicas." (CASTEL, 2004, p. 100). Estas estrategias se basan en la norma de interioridad, es decir, en la modificación de las conductas individuales de los beneficiarios

[...] incitándolos a cambiar sus representaciones y reforzar sus motivaciones para "salir", como si fueran los principales responsables de la situación en la que se encuentran." (CASTEL, 2004, p. 97, grifo do autor). humano y superación de la pobreza intergeneracional

Rosanvallon (1995) observa que el ingreso mínimo de inserción (RMI) revincula de forma inédita los derechos sociales y las obligaciones morales configurando un derecho individualizado por un lado, y un derecho condicionado por el otro, de modo tal que la situación particular y el comportamiento individual quedan en un mismo registro. Seguidamente, se pregunta sobre el carácter de derecho de estas prestaciones en estas condiciones cuando, en esencia, un derecho es de aplicación universal e incondicional. Esta crítica es válida también para nuestros PTRC.

La idea de inversión de la deuda contribuye a pensar estas alteraciones filosóficas de la protección social:

La política social del siglo XX pensaba que como consecuencia de su participación en la vida social, había algunas personas víctimas de accidentes diversos. Accidentes de trabajo, desempleo, una infancia muy dura etc. La sociedad tenía una deuda con esas personas, les debía asistencia, que es lo que en Francia dio derecho, lo que se llama el derecho a la asistencia, un derecho inscripto en la ley. Toda persona tiene derecho a ser asistida. Ahora se invierten las cosas y se convence a la persona que recibe una ayuda de que está en deuda con la sociedad porque no hay ninguna razón que justifique que una persona sea ayudada. La vamos a ayudar para que pueda salir adelante y entonces, como nos debe algo (el dinero que le estamos dando), le vamos a exigir una contrapartida. (MERKLEN, 2013, p. 12).

La denominada inversión de la deuda y la contractualización e individualización de la protección social contenida, fundamental pero no exclusivamente, en los PTRC son parte de una transformación en la orientación filosófica de la protección social sustentada en la retórica de la responsabilidad individual.

En palabras de Rosanvallon (1995, p. 36) "[...] la aceptación de la solidaridad comienza [ahora más que antes] a acompañarse con una exigencia de control de los comportamientos." En ese sentido, plantea que cuando se multiplican las informaciones disponibles sobre los individuos se produce "[...] un movimiento de desolidarización: la información es el alimento de la diferenciación." (ROSANVALLON, 1995, p. 56). El registro de los individuos y el inventario de sus diferencias pretende personalizar la asistencia social con el objetivo de alcanzar una mayor eficiencia:

Es en esta búsqueda de mayor eficiencia donde se encuentran los mayores desafíos del futuro: «personalizar» todavía más la asistencia social [...] Como Banco Mundial, nos complace haber colaborado durante los últimos años en el diseño y en las mejoras implementadas al sistema de protección 
social en Uruguay. (SIEGENTHALER apud BANCO MUNDIAL, 2013).

De este modo,

[...] cuanto mayores y más específicas sean las condiciones que se impongan, cuanto más diferenciadas sean entre grupos de beneficiarios, y cuanto más se vinculen esas condiciones con el comportamiento, más se alejará del campo de los derechos y más la tensión seguridad social/asistencia traccionará hacia la segunda. (HINTZE; COSTA, 2011, p. 176).

Los sistemas de control y vigilancia construidos con el objetivo de verificar el cumplimiento de las condicionalidades permiten introducir un sentido más concreto de la noción de individualización, entendida como la identificación de la población beneficiaria y su incorporación en un registro documental (VECINDAY, 2014). La estigmatización del asistido permanece en estos dispositivos tecnificados de identificación y control: ni las herramientas de selección focalizada ni las de vigilancia pierden su carácter vejatorio. La tecnificación se introduce en el hogar para observar, indagar, clasificar, examinar $y$, eventualmente, castigar.

Así mismo, parece que se perdiera de vista que las sanciones por incumplimiento de las condicionalidades operan como refuerzo de la vulnerabilidad de las familias beneficiarias, y sobre todo, de sus niños y adolescentes. La identificación de un derecho vulnerado por inasistencias escolares o insuficientes controles sanitarios deriva en la posible pérdida de la transferencia agregando a la situación familiar una restricción en sus ingresos, que en los sectores cuanto más pobres son, como es sabido, se ven mayormente afectados por el consumo de alimentos. Esta inconsistencia entre la pretensión declarada de garantizar derechos exigiendo condicionalidades y el efecto práctico de reforzar la vulnerabilidad al sancionar por incumplimiento, es más fuerte aún cuando se observa que son las familias más pobres y vulnerables las que presentan mayores dificultades para cumplir con los comportamientos demandados.

Si bien en la región se registran diferentes grados de control y de aplicación de sanciones por incumplimiento, los PTRC se estructuran a partir de una filosofía conductista (WACQUANT, 2011) montada sobre un sistema donde la prestación monetaria oficia como estímulo a la acción o como sanción ante la inacción u omisión (VECINDAY, 2013).

La relevancia adjudicada en cada caso a los sistemas de control y sanción sobre las condicionalidades revela el papel asignado a las transferencias monetarias y a la activación de capital humano como estrategias asociadas para combatir la pobreza. En otros términos, se puede afirmar que a la jerarquización de la transferencia monetaria le corresponden mecanismos flexibles de control y sanción e, inversamente, al énfasis sobre la inversión en capital humano se asocia con sistemas rígidos de control y sanción. De este modo, mientras que las AFAM-PE y, en menor medida, la $\mathrm{AUH}$ privilegian las condicionalidades como estrategia de reducción de la pobreza a largo plazo afectando, incluso, el papel de la transferencia en el alivio inmediato de la deprivación severa suspendiendo o reteniendo parte del beneficio, el BF se centra en el objetivo de mejorar el acceso al consumo de las familias pobres siendo los comportamientos exigidos por las condicionalidades un logro que no responde solo al estímulo económico sino que requiere de mecanismos de acompañamiento familiar.

Los contextos nacionales ofrecen pistas para comprender los particulares rasgos de los programas en general y, específicamente, respecto a significación de las acciones desencadenadas ante el no cumplimiento. En el caso de Brasil, la magnitud y la masividad de la pobreza y la desigualdad han sido una constante estructural de su desarrollo capitalista y con ese punto de partida parece una estrategia razonable jerarquizar su alivio inmediato frente a la activación de los pobres como forma de interrumpir su reproducción intergeneracional. La comprensión de los factores que explican los problemas de cobertura de las políticas sanitarias y educativas dentro de los sectores de mayor pobreza ha sido clave para promover programas de acompañamiento familiar en situaciones de incumplimiento de las condicionalidades ${ }^{2}$. Por otro lado, Argentina y Uruguay son dos de los países latinoamericanos con mejores indicadores sobre desarrollo humano, distribución del ingreso y pobreza y es el deterioro de los logros educativos y sanitarios, respecto a un pasado reciente, una de las principales preocupaciones depositadas sobre un núcleo duro de la pobreza que persiste pese a la superación de la pobreza e indigencia medida por ingresos. En países que, aún manteniendo problemas de oferta, se han caracterizado por una amplia prestación de servicios, fundamentalmente, educativos y sanitarios, en menor medida, el punto de partida parece situarse en un escalón mayor. En estos casos, las condicionalidades en tanto comportamientos esperados, son privilegiadas al punto de afectar o hacer caer la transferencia monetaria. Sin embargo, llama la atención que ni las AFAM-PE ni la AUH prevén en su diseño mecanismos de apoyo ante situaciones de incumplimiento.

\section{CONCLUSIÓN}

El combate a la pobreza es el objetivo central de los PTRC y si bien, han desempeñado un papel importante en la reducción de la indigencia, menos 
claro es su aporte a la disminución de la pobreza. Esta parece deber su reducción más a las mejoras salariales, a la reducción del desempleo y al combate a la informalidad que al efecto de las transferencias. Este conjunto de medidas ha permitido, sin duda, mejorar las condiciones de vida de ciertos segmentos de la clase trabajadora. Sin embargo, si bien los efectos sobre la indigencia y la pobreza medidas por ingreso son visibles, los atributos duros de la pobreza medidos por Necesidades Básicas Insatisfechas (NBI) no han registrado modificaciones sustantivas. Se podría concluir, entonces, que la reducción de la pobreza parece ser efecto de una coyuntura económica favorable que, sumado a mecanismos regulatorios, alivió los déficits de ingresos en los hogares más pobres que incrementaron su capacidad de consumo. Sin embargo, queda en el debe el rebatimiento de la pobreza y la indigencia en dimensiones que, sin duda, requieren de políticas sostenidas en el tiempo y cuyos efectos no son inmediatos (educación, salud, vivienda, trabajo).

Las prestaciones asistenciales han crecido en calidad, cantidad y cobertura. Sin embargo, la ayuda social tiende a permearse y/o combinarse con componentes y políticas punitivas dirigidas a estas poblaciones. Este componente punitivo está presente en las sanciones previstas por el incumplimiento de las condicionalidades.

Por otro lado, los PTRC operan sobre el valor de uso como mercancia de la futura fuerza de trabajo. Los PTRC se proponen, a largo plazo, incrementar el valor de uso de la fuerza de trabajo invirtiendo en capital humano. Al incrementar la futura productividad de los cuerpos mediante su cuidado por parte del sistema de salud y su calificación a través del sistema educativo su valor de uso crecerá.

Asimismo, los PTRC no se proponen afectar el valor de cambio de la fuerza de trabajo. Es decir, no pretenden suministrar prestaciones a quienes se encuentren en situación de convertir el beneficio en recurso que mejore sus condiciones de negociación salarial. De este modo, las prestaciones vehiculizadas en los PTRC no tienen efecto alguno en el arbitraje de intereses diferenciales de trabajadores y empleadores.

Quizás la principal virtud de las condicionalidades es que nos convoca a pensar estas prestaciones dirigidas a combatir la pobreza en su relación con prestaciones de carácter universal. Es decir, el debate sobre las condicionalidades nos reenvía al análisis de las transferencias en el marco más amplio del repertorio de políticas sociales. En ese sentido, se advierte que la mayor cobertura de estas políticas, posible de atribuir al efecto producido por los PTRC sobre la demanda, no ha sido acompañada de la suficiente ampliación de las capacidades y respuestas estatales. Persisten problemas de calidad de los servicios públicos educativos y sanitarios que no logran ofrecer respuestas adecuadas a la magnitud de los problemas que pretenden atender.

\section{REFERENCIAS}

BANCO MUNDIAL. Hacia un Uruguay más equitativo: los desafíos del sistema de protección social. [S. L.], 2013. Disponible en:<http://www. worldbank.org/content/dam/Worldbank/document/ LAC/hacia_un_uruguay_m as_equitativo_web.pdf $>$. Acceso en: 7 jun. 2015.

BAUMAN, Z. La sociedad individualizada. Madrid: Ediciones Cátedra, 2001

BENTURA, J. P.; VECINDAY, L. ¿Dinero o especies? Los usos del dinero en las estrategias de combate a la pobreza. In: CONGRESO DE LA RED ESPAÑOLA DE POLÍTICA SOCIAL, 5., 2015, Barcelona. Anais... Barcelona: Red Española de Política Social, 2015.

CASTEL, R. La inseguridad social: ¿qué es estar protegido? Buenos Aires: Manantial, 2004. Segunda parte.

DANANI, C. América Latina luego del mito del progreso neoliberal: las políticas sociales y el problema de la desigualdad. Cièncias Sociais Unisinos, São Leopoldo, v. 44, n. 1, jan./abr. 2008.

GIDDENS, A; BECK, U; LASH, S. Modernización reflexiva: política, tradición y estética en el orden social moderno. Madrid: Ed. Alianza, 1997.

HINTZE, S.; COSTA, M. La reforma de las asignaciones familiares 2009: aproximación al proceso político de la transformación de la protección. In: DANANI, C.; HINTZE, S. (Coords.). Protecciones y desprotecciones: la seguridad social en la Argentina 1990- 2010. Buenos Aires: Universidad Nacional de General Sarmiento, 2011.

INSTITUTO CUESTA DUARTE. Informe de Coyuntura trimestral: primer trimestre de 2015. Montevideo, 2015. Disponible en:<http:// cuestaduarte.org.uy/investigacion/economia/ informes-de-coyuntura/item/64-informe-decoyuntura-primer-trimestre-de-2015>. Acceso: 5 maio 2015.

MERKLEN, D. Conversaciones sobre lo público. Revista Debate Público: reflexión de trabajo social, Argentina, n. 6, 2013. Entrevista realizada por la Dra. Claudia Danani.

PIEDRA, L. Transferencias en especie versus transferencias en metálico: modelos empíricos. In: ENCUENTRO DE ECONOMÍA PÚBLICA, 11., 2004, Barcelona. Anais... Barcelona, 2004. 
ROSANVALLON, P. La nueva cuestión social: repensar el Estado providencia. Buenos Aires: Ediciones Manantial, 1995.

SEN, A. Desarrollo y libertad. Buenos Aires: Grupo Planeta, 2000.

SILVA, M. O. da S. e; GUILHON, M. V. M.; LIMA, V. F. S. de A. As Condicionalidades e o Índice de Gestão Descentralizada (IGD) enquanto Dimensões Centrais do Bolsa Família (BF): uma incursão na realidades do programa no Maranhão. Cadernos de Pesquisa, São Luís, ano 1, n. 1, 2013. Disponível em:<www.gaepp.ufma.br>. Acceso: 6 maio 2015.

VECINDAY, L. Transformaciones institucionales y tecnológicas del esquema de protección social: el caso del Plan de Centros de Atención a la Infancia y la Familia en el Uruguay. Montevideo: Comisión Sectorial de Investigación Científica, Universidad de la República, 2014.

La reconfiguración punitiva del tratamiento social de la pobreza en el Uruguay actual. Textos y Contextos, Porto Alegre, v. 12, n. 2, 2013.

WACQUANT, L. Forjando el Estado neoliberal: workfare, prisonfare e inseguridad social. Revista Prohistoria, Rosario, n. 16, jul./dic. 2011.

\section{NOTAS}

1 Esta ponencia, presentada en la VII Jornada Internacional de Políticas Públicas, São Luís, 25 a 28 de agosto de 2015, contiene resultados parciales de estudios desarrollados con apoyo de la Fundação Coordenação de Aperfeiçoamento de Pessoal de Nível Superior (CAPES) y del Conselho Nacional de Desenvolvimento Científico e Tecnológico (CNPq).

2 Es indudable que este acompañamiento familiar responde solo a uno de los factores que explican los problemas de acceso a las prestaciones sanitarias y educativas dejando fuera, por ejemplo, el problema nada menor de la oferta y calidad de los servicios ofrecidos.

Maria Laura Vecinday Garrido

Asistente Social

Doctora en Ciencias Sociales en Facultad Latinoamericana de Ciencias Sociales (FLACSO)

Docente e investigadora del Departamento de Trabajo

Social de la Facultad de Ciencias Sociales de la

Universidad de la República (UDELAR)

E-mail: lauravecinday@gmail.com

Universidad de la República - UDELAR

Oficinas Centrales

Av. 18 de Julio 1968, Montevideo/Uruguay 